\title{
RF Wireless Power Transfer for EIT Neonate Lung Function Monitoring
}

\author{
Mandana Ardeshir Tanha ${ }^{1}$, Farnaz Fahimi Hanzaee ${ }^{1}$, Richard Bayford ${ }^{2}$, and Andreas Demosthenous ${ }^{1}$ \\ ${ }^{1}$ Department of Electronic and Electrical Engineering, University College London, Torrington Place, London WC1E 7JE, UK \\ ${ }^{2}$ Department of Natural Sciences, Middlesex University, London NW4 4BT, UK \\ e-mail:m.tanha.12@ucl.ac.uk; a.demosthenous@ucl.ac.uk
}

\begin{abstract}
This paper presents an antenna-based RF wireless power and data transfer system for wireless neonate lung function monitoring in an intensive care unit using electrical impedance tomography (EIT). The proposed dual-band system comprises a directional slotted patch transmit antenna and a slotted flexible omnidirectional monopole antenna operating at 2.51 GHz and 5.1 GHz. A slotting technique unique to each antenna is proposed to achieve dual-band operation and size miniaturization for each antenna. Also, the arraying technique combined with parasitic elements is employed to increase the receive antenna gain and in turn the received power level. Both transmit and receive antennas were simulated and fabricated. Following FCC safety regulations, measurements show $20 \mathrm{~mW}$ received power when the receiver and the transmitter are spaced $19 \mathrm{~cm}$ apart.
\end{abstract}

Keywords - Antenna, dual band, electrical impedance tomography (EIT), slotting technique, wireless power transfer.

\section{INTRODUCTION}

Continuous pulmonary function monitoring of neonates in intensive care plays an important role in the evaluation of lung function status and for providing an accurate clinical diagnosis and management. Electrical impedance tomography (EIT) is a non-invasive technique which can be used for continuous lung function monitoring of premature new-borns [1]. By means of EIT and injecting low frequency electrical currents through a pair of electrodes the electrical resistivity variation of the lung tissue can be measured. To produce an image of neonate lung, a wearable EIT based belt with the multiple electrodes placed around the belt is envisioned [2]. The belt structure is designed using a flexible substrate and the EIT electronic circuits would ideally be printed on the flexible belt. Supplying electrical power to the EIT circuits is a critical issue due to the very sensitive nature of premature infants under intensive care.

One basic approach is to use batteries; however, they must be replaced or recharged over the monitoring period which can be up to 72 hours. Also, cables and wires required for battery connection would make the wearable device uncomfortable for the neonate. Thus, wireless power transfer (WPT) can be an ideal non-invasive solution for this application. WPT has been extensively used to supply power for biomedical applications. Inductive link coupling is a popular technique for various biomedical applications [3]-[4]. It comprises a primary coil at the transmitter and a coupled secondary coil at the receiver to transfer energy. The inductive link has a simple structure, and it is easy to fabricate. In the case where the inductive link is designed for wearable biomedical applications printed spiral coils may be used. Despite these advantages, an inductive link based WPT is only an effective technique for short range applications (typically less than $2 \mathrm{~cm}$ distance) and misalignments between the primary and secondary coils can significantly reduce the mutual

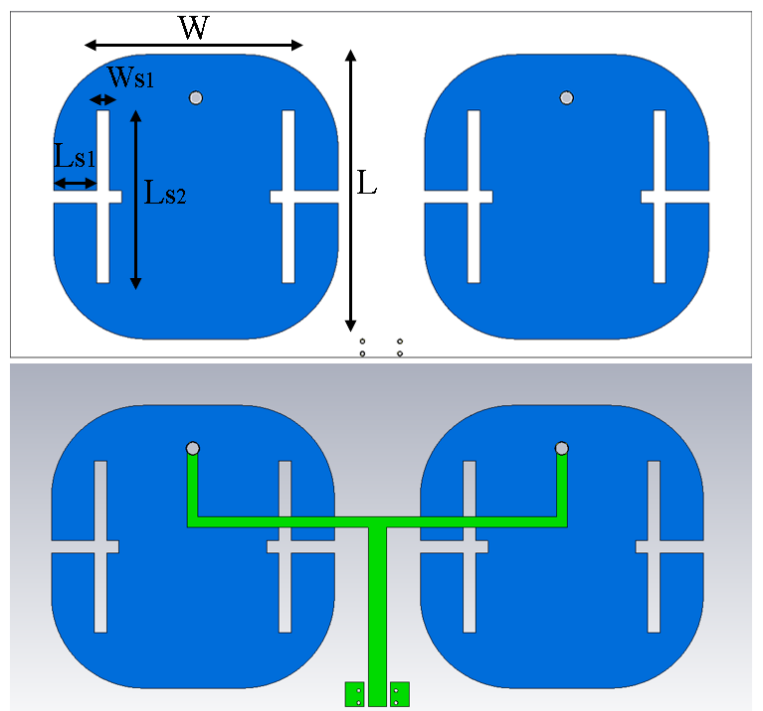

Fig.1. Slotted transmit patch antenna array with parallel power distribution network.

inductance which results in low power transfer efficiency and power delivered to the load. To overcome the limitations of the inductive link WPT, RF antenna based WPT can be designed [5]-[8]. This type of WPT system is composed of a transmit antenna and a receive antenna (e.g. at the wearable device). RF WPT can be used for long range biomedical applications. Specifically, for neonate lung monitoring the transmitter antenna can be placed underneath the bed on the floor and the receive antenna can be printed on a flexible wearable belt. Similar to an inductive link based WPT system, the misalignment between the receiver and transmitter antennas reduces the delivered power. However, this can be effectively compensated by controlling the radiation pattern and the gain of the receive antenna. Moreover, designing a dual-band antenna allows both data and power transmission. In RF WPT systems, higher data transmission can be achieved due to high frequency of operation.

The dual-band antennas are widely used for RF WPT systems to transmit both power and data using a single antenna at each side of the power transfer link. The main challenge is to devise a technique to design a compact antenna with dualband antenna operation. The antenna bands of operation can be controlled and excited by designing various feeding networks based on techniques such as co-planar wave guide [9] and aperture coupling [10]. However, using such techniques increases the antenna complexity and fabrication cost. The dual antenna operation can also be excited by designing a meta-surface- based antenna [11], using stacking techniques [12] and dual antenna feed ports [13]. These techniques add to antenna design complexity and increases the 


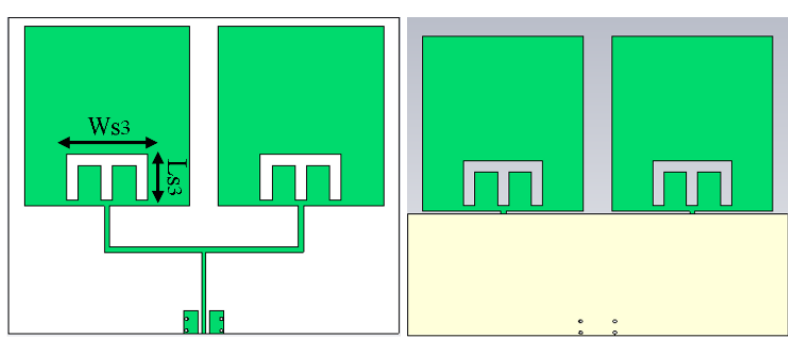

Fig. 2. Slotted receive monopole antenna with parallel feeding network.

antenna physical size which is not desirable for neonate wearable biomedical applications.

The antenna dual-band operation can be also obtained using slotting technique. By loading slots with optimised length and position into the antenna structure based on antenna surface current distribution and fundamental mode, a dual resonance can be achieved. This technique enables the antenna physical dimension to be reduced, and its bands of operation can be controlled independently. Various slotting techniques for designing receive antennas for RF WPT and implantable medical devices have been reported in the literature such as slotted planar inverted-f antenna [5] and slotted antenna based on quasi-Yagi antenna [14]. However, in these slotted structures a thick rigid substrate is used to achieve the desirable antenna performance. Based on the antenna fundamentals a thick substrate is desirable to achieve high antenna performance (i.e. wide bandwidth). However, the flexible materials which are used for wearable biomedical devices are thin by nature to allow flexibility and designing a high-performance antenna on such a substrate with thickness less than 5 mil is very challenging.

In this paper a WPT system consisting of a dual band slotted transmit antenna on a rigid substrate and a slotted receive antenna on a thin ( 3 mil) flexible substrate is proposed for neonate pulmonary function monitoring using EIT. Two different slotting techniques are proposed specific to each of the transmit and receive antennas to miniaturize their physical size and to achieve dual band operation using only a single feeding port for each antenna. The receive slotted antenna is designed on a thin flexible substrate. The parasitic elements on top of the radiating elements combined with the array technique is proposed to increase the gain of the receiver antenna which results in higher delivered power. Both antennas were fabricated, and the RF link power transfer was measured. The rest of the paper is organized as follows. In Section II methods and fundamentals of designs are discussed. Section III presents simulations and measurements of the prototype antennas and power transfer link. Concluding remarks are in Section IV.

\section{METHOD AND MATERIAL}

\section{A. WPT Structure}

The proposed wireless power transfer system consists of a dual band directional slotted patch antenna at the transmitter and a dual band omnidirectional slotted monopole antenna at the receiver, as shown in Fig 1 and Fig 2. Both antennas were designed to resonate at $2.5 \mathrm{GHz}$ and $5 \mathrm{GHz}$. The lower band of frequency is used to transmit power and the higher band of frequency to transmit data. The antenna with directional radiation pattern at the receiver is designed to illuminate the area of interest where the receiver antenna is positioned. Also,

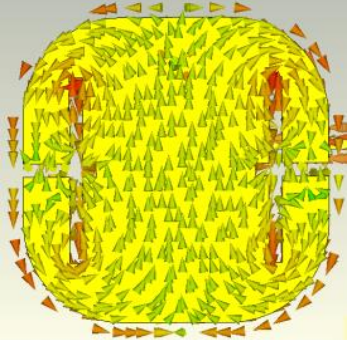

(a)

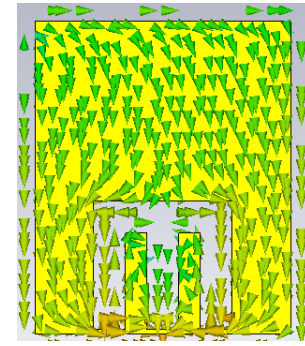

(b)
Fig. 3. (a) Slotted transmit patch antenna current distribution at $2.51 \mathrm{GHz}$, and (b) slotted receive monopole antenna current distribution at $2.51 \mathrm{GHz}$.

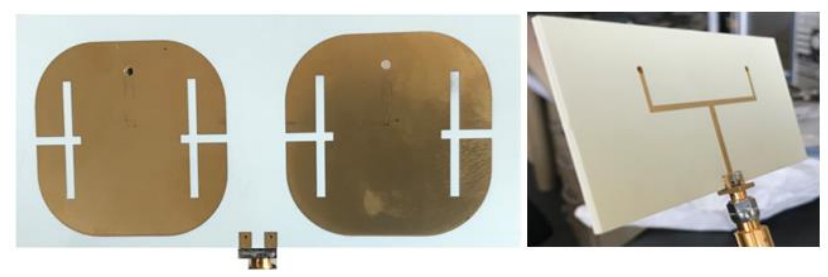

Fig. 4. Fabricated slotted transmit antenna.

the antenna with omnidirectional pattern at the receiver can mitigate for misalignments between the transmit and receive antennas. In practice, the misalignments would mainly be caused by neonate movements.

\section{B. Transmit Antenna}

The designed transmit antenna is a slotted multilayer structure. The antenna structure is composed of two stacked RO4350B rigid laminates with 3.66 dielectric constant. The thickness of the top layer substrate on which the antenna radiating element is positioned is $1.575 \mathrm{~mm}$. The antenna microstrip feed lines are placed on the bottom substrate with $0.787 \mathrm{~mm}$ thickness. The thick substrate is used for the radiating elements to improve the antenna bandwidth and radiation. and the thin substrate is used for the transmission lines to avoid spurious radiation. In this case, a ground plane is positioned in the middle between the top and bottom substrates which can further isolate the antenna radiating element and feed network. The radiating elements are connected to the feed network using hollow metallic vias. To avoid shorting the feed network and radiating elements to the ground, isolation pads were designed on the ground plane. The dimension of the pad is optimised to achieve high impedance matching. The dual band antenna operation has been achieved by means of loading corrugation and slots into the patch antenna structure based on the antenna current distribution. By means of optimising the position and the shape of corrugations and slots the current path on the antenna and the excitation modes is controlled. The slotting technique is also used to miniaturise the size of the antenna which is crucial for this application. To further enhance the gain of the transmit antenna, the arraying technique has been used. Based on above, a $2 \times 1$ antenna array has been designed for this application.

The proposed slotting technique is unique to the antenna structure. Cross shaped slots were loaded into both of the nonradiating edges of the patch antenna. The slots are identical and symmetrical to achieve a uniform directional radiation pattern. The position, length, and width of the slots were optimised to achieve the desired performance. The slots positioned along the direction of the current on the patch 

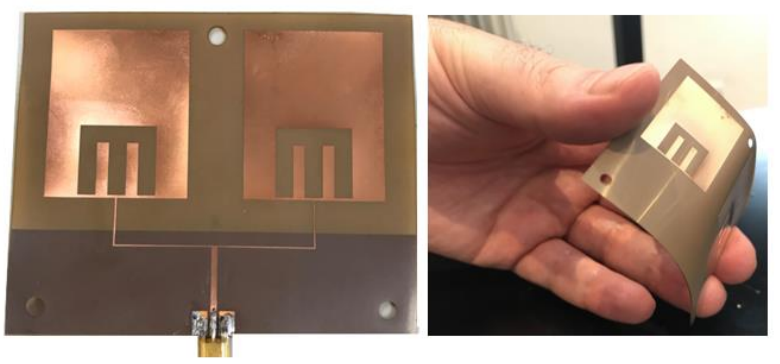

Fig. 5. Fabricated slotted receive antenna array and the slotted parasitic elements.

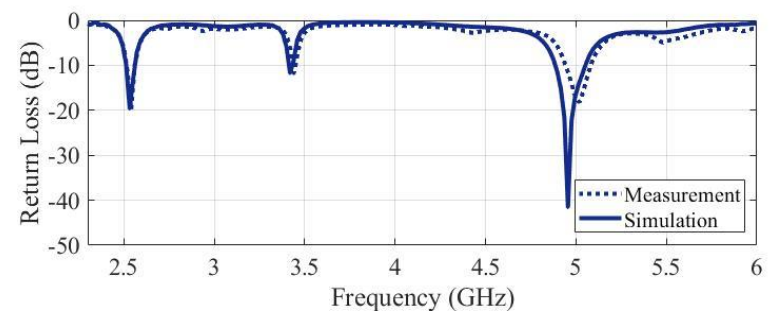

Fig . 6. Slotted transmit patch antenna return loss.

antenna are to excite lower frequency modes. This is due to the fact that the current travels a longer path. On the other hand, the slots positioned perpendicular to the direction of the current are to excite high frequency modes. Fig 3(a) shows the slotted antenna current distribution at $2.51 \mathrm{GHz}$ and the current paths introduced to the antenna by loading slots. The transmit antenna optimised slot parameters are $\mathrm{W}=0.15 \lambda_{0}$ $\mathrm{mm}, \mathrm{W}_{\mathrm{s} 1}=0.016 \lambda_{0} \mathrm{~mm}, \mathrm{~L}=0.3 \lambda_{0} \mathrm{~mm}, \mathrm{~L}_{\mathrm{s} 1}=0.05 \lambda_{0} \mathrm{~mm}$, and $\mathrm{L}_{\mathrm{s} 2}=0.2 \lambda_{0} \mathrm{~mm}$. The antenna was fabricated based on these parameters.

\section{Recieve Antenna}

The proposed receive antenna is a single layer slotted structure designed on a flexible polyimide Dupont AP8535 with $0.0762 \mathrm{~mm}$ thickness and 3.5 dielectric constant. The slotting technique has also been used to achieve antenna miniaturisation and dual band operation by optimising and positioning the slots in the structure of the antenna. The resulting printed slotted monopole antenna with partial ground plane has an omnidirectional radiation pattern. In neonate vital sign monitoring application, a high level of received power is desirable at the receiver to supply sufficient power to the electronic circuits on the wearable flexible belt. However, RF safety considerations which will be discussed in the next section may limit the received power. By increasing the transmit power level and the transmit antenna gain the distance between the receiver and the transmitter may be increased to adhere to the RF safety regulations. Hence, the received power level cannot be enhanced solely by using the techniques described.

A technique which can be used to increase the received power level is to control and enhance the gain of the receive antenna. Based on antenna theory [15], by increasing the gain of the antenna, the antenna directivity increases, and the antenna beamwidth narrows down. As a result, the antenna radiation pattern cannot be perfectly omnidirectional. Therefore, technical trade-offs should be considered between the receive antenna gain, transmit antenna gain, and the transmit power level to achieve the desired performance and
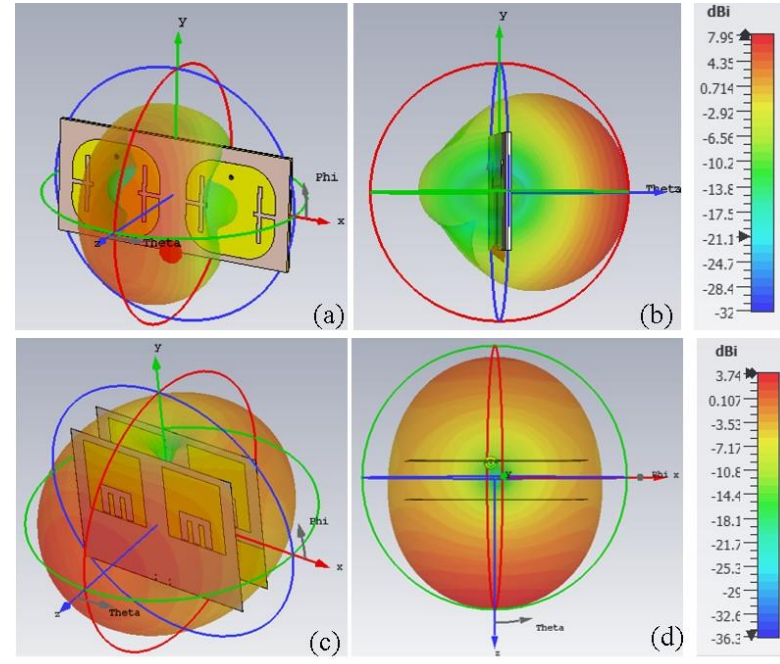

Fig . 7. 3D radiation pattern. (a) and (b) The transmit antenna directional radiation pattern at $2.5 \mathrm{GHz}$, (c) and (d) the receive antenna semiomnidirectional radiation pattern at $2.5 \mathrm{GHz}$.

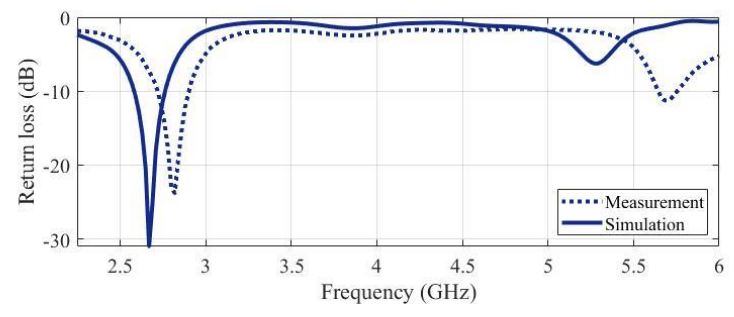

Fig. 8. Slotted receive monopole antenna return loss.

the required amount of delivered power. It should be noted that in the case of a fixed given transmit power level for the transmit antenna, and safe distance between the antennas, the receive antenna gain can be increased as required.

In this application two techniques have been combined to increase the gain of the receive antenna namely arraying technique and using parasitic elements in the proximity of the slotted radiating element. In the arraying technique by adding every single element to the array structure the antenna gain increases by $3 \mathrm{~dB}$. Theoretically, the overall array gain is defined as the addition of the array factor and the single element antenna gain. The array factor can be also estimated by taking the logarithm of the number of elements $((10 \log N)$ where $N$ is the total number of array elements). Also, by using passive parasitic elements which are exactly identical to the receive array radiating elements the antenna gain can be improved. The parasitic elements are not electrically connected to the antenna, and their position is optimised to achieve high performance. The mutual coupling between the antenna elements and the parasitic elements and variations in the antenna current distribution leads to antenna gain enhancement.

A slotting technique is also proposed to design the receive antenna. Similar principles used to design the transmit antenna were employed to design the receive antenna. In this design, an $\mathrm{M}$ shaped slotting technique is proposed. The position and parameters of the proposed slotting technique are also optimised to achieve high performance. Fig 3(b) shows the slotted antenna current distribution at $2.51 \mathrm{GHz}$ and the current paths introduced to the antenna by loading slots. The antenna parameters were optimised by parameter sweeping. 


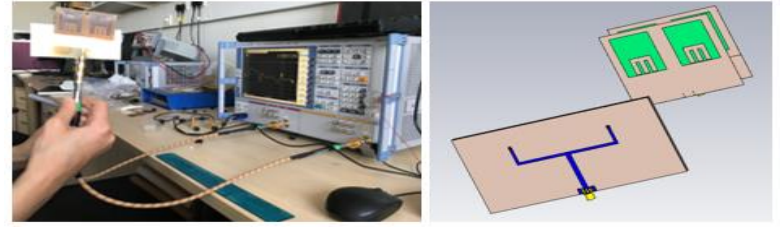

Fig. 9. Proposed antenna based WPT measurement and simulation setup.

The receive antenna parameters are $\mathrm{W}_{\mathrm{s} 3}=0.1 \lambda_{0} \mathrm{~mm}, \mathrm{~L}_{\mathrm{s} 3}=$ $0.1 \lambda_{0} \mathrm{~mm}$. CST Studio2020 was used to develop a finite element model and optimise both the transmit and receive antennas.

\section{Safety Regulations and Link Budget}

The wireless power link was designed to operate at the 2.5 $\mathrm{GHz}$ ISM band. As discussed, the received power level is a function of the antenna characteristics, transmit power level, and path loss. The effective isotropic radiated power (EIRP) is a parameter which should be also considered when analysing the WPT system link budget, Equation (1), where $P_{t}$ is the transmit power and $G_{t}$ is the transmit antenna gain. According to FCC safety regulations, the maximum EIRP should not exceed $36 \mathrm{dBm}$ when the transmit power level is set to $30 \mathrm{dBm}$. The EIRP level can be increased to more than $36 \mathrm{dBm}$ if for each $3 \mathrm{~dB}$ increase in the transmit antenna gain, the transmit power level reduces by $1 \mathrm{~dB}$.

The received power level can be defined based on Equation (2) where $G_{r}$ is the receive antenna gain and $P_{r}$ is the receive power level. The other important parameter in designing a safe RF WPT system is the maximum permissible exposure (MPE) which is defined in Equation (3) [16]. The RF WPT link was designed considering the safety regulations. This is particularly important since the designed WPT is intended for use in neonate intensive care units. The link was designed to achieve approximately $37 \mathrm{dBm}$ EIRP by designing a transmit antenna with $7.9 \mathrm{dBi}$ gain. In this case the transmit power was set to $29 \mathrm{dBm}$ to conform to the safety

$$
\begin{gathered}
E I R P(d B m)=P_{t}(d B m)+G_{t}(d B i) \\
P_{r}(d B m)=E I R P(d B m)+G_{r}(d B i) \\
W_{f}\left(w / m^{2}\right)=\frac{E I R P}{4 \pi d^{2}} \leq 10\left(w / m^{2}\right)
\end{gathered}
$$

regulations. The distance between the transmit and receive antenna is also defined based on the allowed MPE which in this case is about $19 \mathrm{~cm}$. Theoretically, by taking into account of losses, up to $25 \mathrm{~mW}$ can be delivered with this system.

\section{RESULTS}

The transmit and receive slotted antennas were fabricated and measured, as shown in Fig. 4 and Fig.5. The measurement setup was composed of the fabricated transmit and receive antennas, a vector network analyser (VNA) and RF cables. The transmit and receive antennas were fed through an end launcher SMA connector and a coplanar SMP connector, respectively. The RF cables were also used to connect each antenna to a single port of the VNA. Using this setup and calibrating the RF cables, the S-parameters were measured for

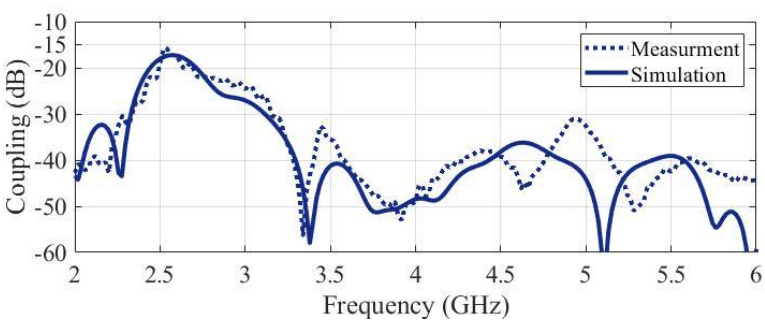

Fig. 10. The coupling $\left(S_{21}\right)$ between the transmit and receive antennas , distanced by $19 \mathrm{~cm}$.

each antenna and the coupling between the receive and transmit antennas.

The transmit antenna return loss (i.e. $\mathrm{S}_{11}$ ) is shown in Fig. 6. Antenna dual band operation was achieved. There is also a resonance at $3.4 \mathrm{GHz}$ which should be supressed by means of filtering and slightly changing the antenna parameters in the next version of the antenna. The transmit antenna has a directional radiation pattern with $7.9 \mathrm{dBi}$ gain as shown in Fig. 7 (a) and (b). The receive antenna return loss is shown in Fig8. The slight frequency shift between the simulation and measurement results mainly at the higher band of frequency may be due to fabrication tolerances and soldering effects, however, the antenna is still operating at the desired frequency band for power transfer which is the main focus of this paper. The simulated radiation pattern of the slotted received antenna with parasitic elements is shown in Fig 7(c) and (d). In this case the parasitic elements are distanced $15 \mathrm{~mm}$ at the top of the radiating elements. As expected, the achieved antenna radiation pattern is semi-omnidirectional with $3.74 \mathrm{dBi}$ gain.

The power transfer link was setup using the fabricated antennas for initial measurements of received power, as shown in Fig. 5. In this measurement setup the slotted transmit and receive antennas were separated by $19 \mathrm{~cm}$ following FCC safety regulations considering the transmit antenna gain, transmit power level, and EIRP. The transmission coefficient (i.e. $\mathrm{S}_{21}$ ) between the transmit antenna was measured which was in agreement with the simulation results. As Fig.10 shows that the delivered power in this setting is approximately -17 $\mathrm{dB}(\sim 20 \mathrm{~mW})$ at $2.5 \mathrm{GHz}$.

\section{CONCLUSION}

In this paper a slotted patch antenna and a slotted monopole antenna have been presented for RF WPT for use in a wearable EIT neonate vital sign monitoring device. The antennas have been designed to resonate at $2.5 \mathrm{GHz}$ for wireless power transfer and $5.1 \mathrm{GHz}$ for data telemetry. The simulation and measurement results of the fabricated antennas have been shown to be in agreement. The wireless power transfer link has been tested using the fabricated transmit and receive antennas. Following FCC safety regulations initial measurements showed that up to $20 \mathrm{~mW}$ can be received.

\section{REFERENCES}

[1] Wu, Y., Jiang, D., Bardill, A., Bayford, R., and Demosthenous, A. (2019). "A $122 \mathrm{fps}, 1 \mathrm{MHz}$ bandwidth multi-frequency wearable EIT belt featuring novel active electrode architecture for neonatal thorax vital sign monitoring." IEEE Transactions on Biomedical Circuits and Systems, 13(5), 927-937.

[2] Wu, Yu, et al. "Live Demonstration: A wearable torso shape detection belt for lung respiration monitoring." 2019 IEEE International Symposium on Circuits and Systems (ISCAS). IEEE, 2019. 
[3] Kiani, Mehdi, Uei-Ming Jow, and Maysam Ghovanloo. "Design and optimization of a 3-coil inductive link for efficient wireless power transmission." IEEE Transactions on Biomedical Circuits and Systems 5.6 (2011): 579-591.

[4] Schormans, Matthew, Virgilio Valente, and Andreas Demosthenous. "Practical inductive link design for biomedical wireless power transfer: A tutorial." IEEE Transactions on Biomedical Circuits and Systems 12.5 (2018): 1112-1130.

[5] Bakogianni, Sofia, and Stavros Koulouridis. "A dual-band implantable rectenna for wireless data and power support at sub-GHz region." IEEE Transactions on Antennas and Propagation 67.11 (2019): 6800-6810.

[6] Sundarsingh, Esther Florence, et al. "Polygon-shaped slotted dual-band antenna for wearable applications." IEEE Antennas and Wireless propagation letters 13 (2014): 611-614.

[7] Bahrami, Hadi, et al. "Biological channel modeling and implantable UWB antenna design for neural recording systems." IEEE Transactions on Biomedical Engineering 62.1 (2014): 88-98.

[8] Ding, Zhiguo, et al. "Application of smart antenna technologies in simultaneous wireless information and power transfer." IEEE Communications Magazine 53.4 (2015): 86-93.

[9] Wong, Kin-Lu, et al. "Very-low-profile grounded coplanar waveguidefed dual-band WLAN slot antenna for on-body antenna application." IEEE Antennas and Wireless Propagation Letters 19.1 (2019): 213217.
[10] Sharma, Anand, et al. "Novel asymmetrical Swastik-shaped aperture coupled cylindrical dielectric resonator antenna with dual-band and dual-sense circular polarization characteristics." Microwave and Optical Technology Letters 61.2 (2019): 405-411.

[11] Zheng, Qi, et al. "Dual-band metasurface-based CP low-profile patch antenna with parasitic elements." IET Microwaves, Antennas \& Propagation 13.13 (2019): 2360-2364.

[12] Yang, HongCai, Yi Fan, and XiongYing Liu. "A compact dual-band stacked patch antenna with dual circular polarizations for BeiDou navigation satellite systems." IEEE Antennas and Wireless Propagation Letters 18.7 (2019): 1472-1476.

[13] Kumari, Tripta, et al. "Dielectric resonator based two-port dual band antenna for MIMO applications." International Journal of $R F$ and Microwave Computer-Aided Engineering 29.12 (2019): e21985.

[14] Shi, Yanyan, et al. "An efficient broadband slotted rectenna for wireless power transfer at LTE band." IEEE Transactions on Antennas and Propagation 67.2 (2018): 814-822.

[15] Balanis, Constantine A. Antenna theory: analysis and design. John Wiley \& Sons, 2016.

[16] Bercich, Rebecca A., Daniel R. Duffy, and Pedro P. Irazoqui. "Far-field RF powering of implantable devices: Safety considerations." IEEE Transactions on Biomedical Engineering 60.8 (2013): 2107-2112. 